\title{
La diversité culturelle et linguistique comme ressource à la créativité
}

Enrica Piccardo, Oise - Université de Toronto

\section{Introduction}

Le processus de globalisation s'accompagne de phénomènes de mobilité et de changement qui intéressent tous les domaines sociétaux et qui dessinent un paysage sociologique dynamique marqué par la pluralité et la diversification. Les sociétés post-industrielles sont caractérisées par un haut degré de mobilité qui se manifeste non seulement sous la forme de vagues migratoires mais aussi dans le monde du commerce, de la finance, de l'échange d'information et dans tous les domaines de la connaissance. Les sociétés expérimentent constamment des phénomènes que nous pourrions définir avec Jean-Jacques Defert de «détérritorialisation » et de « reterritorialisation » (314). La modernité et le changement concernent chaque aspect de notre vie individuelle et collective, comme le font remarquer Stephen Castles et Mark J. Miller. Nous assistons à une augmentation exponentielle de la diversité qui implique aussi un changement et un renouvellement des formes linguistiques et culturelles. Aucune langue n'est exclue de ce processus, car les immigrants apportent avec eux une quantité de langues et de cultures qui entrent en contact avec les langues et les cultures des sociétés d'accueil et qui agissent comme autant d'éléments catalyseurs de changement (Piccardo, «Plurilingualism »)

Dans le domaine de l'éducation, il n'est pas rare de se retrouver face à des classes où chaque élève, chaque étudiant a, à son actif, une expérience de vie caractérisée par une pluralité des langues et de cultures. En général, la notion de superdiversity (Vertovec) est de plus en plus utilisée pour souligner l'amplitude de ces phénomènes. De leur côté, les politiques, les éducateurs et les chefs d'entreprise, aussi bien dans le monde occidental que dans les pays asiatiques, se rendent de plus en plus compte que la créativité et l'innovation sont deux composantes essentielles du succès économique (Sawyer, Explaining) : les pays passent d'économies industrielles à économies de la connaissance créatrice où on produit des idées plutôt que des objets. Si ceci est le contexte actuel, la question suivante se pose : face à cet accroissement de la diversité culturelle et linguistique, dans quelle mesure la créativité peut-elle être utilisée comme une ressource pour favoriser et valoriser le plurilinguisme au sein d'une société reconnue comme multilingue? 
La réaction sociétaire face à des changements de cette ampleur est différenciée, se situant le long d'un continuum qui va de l'acceptation au rejet, ou encore, pour l'exprimer de manière plus nuancée, d'un intérêt actif vers la différence à une folklorisation qui ne garde que des simulacres de différence.

En fait, on pourrait dire qu'on est confrontés à plusieurs continua selon que l'on se focalise sur l'un ou l'autre des phénomènes liées à cette mutation. Pour ce qui est des langues, on va de la valorisation du multi/plurilinguisme au choix d'utilisation d'une lingua franca; pour ce qui est des cultures, on trouve d'un côté un intérêt actif vers la différence culturelle et de l'autre une générale standardisation des cultures accompagnée de phénomènes de globalisation qui gardent des simulacres de différenciation culturelle (un peu à la façon de McDonald's, qui sert une vinaigrette à base d'huile d'olive et vinaigre balsamique en Italie, et une formule petit café accompagné d'un macaron à Paris).

À partir de ce constat, cette contribution s'interroge sur les implications des différents choix sur le développement de la créativité. Il sera question notamment d'analyser comment la notion de plurilinguisme a permis de créer progressivement une vision plus large et plus valorisante de la diversité en la transformant d'obstacle à opportunité. Ceci nous permettra de nous interroger sur les liens possibles entre plurilinguisme et créativité. En fait, grâce à sa nature multiple et flexible qui valorise toute forme de métissage et d'hybridité et qui permet d'accroitre la prise de conscience de l'unicité de chaque trajectoire individuelle, le plurilinguisme peut créer des conditions favorables à la créativité. À son tour, la créativité, en tant que capacité transversale qui favorise la flexibilité cognitive, constitue une (res)source puissante pour valoriser la diversité linguistique et culturelle au sein de nos sociétés.

\section{Diversité linguistique et vision plurilingue : vers un changement de paradigme}

Dans le domaine de la diversité culturelle, le changement de vision commence en 1962, année charnière, avec la publication d'une recherche de Elizabeth Peal et Wallace E. Lambert qui, en étudiant des enfants bilingues de dix ans, trouve qu'ils ont de meilleures facultés de résoudre des problèmes (problem-solving skills) de pensée abstraite (abstract thinking skills) de construction conceptuelles (higher concept-formation skills) et une plus grande flexibilité mentale (overall higher mental flexibility). L'affirmation de Peal et Lambert selon lesquels l'enfant est « a youngster whose wider experiences in two cultures have given him advantages which a 
monolingual does not enjoy » (20) est destinée à devenir un tournant décisif dans la relation de la communauté scientifique avec la diversité des langues.

Avant cette recherche, la supériorité des monolingues sur les bilingues n'était pas mise en question (Darcy ; Jensen ; Peal et Lambert ; Hakuta et Diaz), car le bilinguisme était considéré comme négatif pour l'intelligence. Le pouvoir intellectuel d'un enfant bilingue serait divisé par deux : on pensait en fait qu'une deuxième langue réduirait l'espace disponible pour d'autres poursuites intellectuelles.

Néanmoins, malgré cette recherche importante et d'autres qui suivirent (Ianco-Worrall ; Hakuta et Diaz), la mentalité peine à changer et, comme le rappelle Colin Baker, la vision monolingue était encore dominante au moment où il écrivait son livre, en 1988. La force du mythe monolingue s'explique par des facteurs historiques, sociologiques, linguistiques et psychologiques. La construction des états-nations du seizième siècle au dix-neuvième siècle, avec son idéologie (un état-une langue, un citoyen-une langue, dans des frontières bien définies), y joue un rôle clé.

De son côté, la constitution de la linguistique comme science avec la distinction de Saussure entre 'langue' et 'parole' et celle entre competence et performance proposée par Noam Chomsky, qui se réfère toujours à un ideal native speaker véhicule inévitablement un message de pureté linguistique, une vision platonicienne de langue comme entité « parfaite ». Dans le domaine de l'enseignement, on assiste au passage d'une vision de l'apprentissage de la langue comme un exercice intellectuel, reproduisant le rôle autrefois joué par l'apprentissage des langues classiques, le latin et le grec, à une vision fortement influencée par les théories psychologiques du behaviorisme qui soulignent le rôle central de l'acquisition d'automatismes et qui nécessitent que les langues soient gardées très séparées. Et il faut attendre la fin des années soixante-dix et l'arrivé de l'approche communicative pour que les choses commencent à changer.

Ce n'est donc que lentement, au cours de la seconde moitié du vingtième siècle, que l'intérêt pour le bilinguisme d'abord et pour le multilinguisme ensuite commence à faire sa percée et les études se multiplient, même si la présence encombrante du parlant natif idéal tel qu'il a été évoqué par Chomsky est encore percevable, car la pensée commune, et pour partie celle scientifique, peine à se libérer d'une disposition monolingue («monolingualer Habitus ») (Gogolin) généralisée. Souvent, en fait, le bilinguisme et le multilinguisme sont réduits à une simple collection de monolinguismes. 
Dans les années récentes, néanmoins, on a assisté à un foisonnement de nouvelles notions qui ouvrent sur de nouveaux horizons. Dans le monde anglo-saxon, des notions entièrement nouvelles ont fait leur percée, telles que translanguaging (Garcia), code-meshing (Canagarajah), multi-competence (Cook), polylingualism (Jørgensen), and transidiomatic practices (Jacquemet) qui ouvrent une vaste gamme de possibilities à la recherche sur la pluralité linguistique. De leur côté, Sinfree Makoni et Alistair Pennycook affirment haut et fort le caractère inventé, construit, artificiel de la Langue, qui finit par devenir un objet mythique et mythisé, et avec elle l'invention du parlant natif idéal et idéalisé et prônent la nécessité de des-inventer les langues.

Mais c'est surtout en Europe, où la familiarité avec une pluralité des langues est plus ancienne et plus répandue, que les notions se précisent. En particulier, une définition innovante et fédératrice a été proposée, celle de plurilinguisme (Coste, Moore et Zarate) comme distinct du multilinguisme. Cette notion reprise et précisée dans le Cadre européen commun de référence pour les langues (CECR) est devenue un des piliers de la nouvelle vision des langues et de leur enseignement prônée par Conseil de l'Europe ( $\mathrm{CoE}$; Piccardo et al ; Canagarajah et Liynage ; Piccardo, «Plurilingualism », « Impact »; North).

Nous allons regarder de plus près la distinction entre plurilinguisme et multilinguisme proposée par le CECR car elle va nous permettre de mieux faire le lien entre pluralité des langues et créativité. Le multilinguisme est défini comme « la connaissance d'un certain nombre de langues ou la coexistence de langues différentes dans une société donnée » (CECR 11). Il n’y a aucune focalisation sur la relation entre les langues. En fait, on spécifie tout de suite après que le multilinguisme est assez facile à atteindre aussi bien au niveau social, avec des communautés qui vivent l'une à coté de l'autre sans contact ou presque, que dans l'éducation, où il suffit de rajouter des langues au curriculum.

Dans le plurilinguisme, au contraire, le principe de relation est central :

$\mathrm{Au}$ fur et à mesure que l'expérience langagière d'un individu dans son contexte culturel s'étend de la langue familiale à celle du groupe social puis à celle d'autres groupes (que ce soit par apprentissage scolaire ou sur le tas), il/elle ne classe pas ces langues et ces cultures dans des compartiments séparés mais construit plutôt une compétence communicative à laquelle contribuent toute connaissance et toute expérience des langues et dans laquelle les langues sont en corrélation et interagissent. Dans des situations différentes, un locuteur peut faire appel avec souplesse aux différentes parties de cette compétence pour entrer efficacement en communication avec un interlocuteur donné. (11) 
Cela est exemplifié de la manière suivante :

Des partenaires peuvent, par exemple, passer d'une langue ou d'un dialecte à l'autre, chacun exploitant la capacité de l'un et de l'autre pour (1) s'exprimer dans une langue et comprendre l'autre. D'aucun peut (2) faire appel à sa connaissance de différentes langues pour comprendre un texte écrit, voire oral, dans une langue a priori «inconnue », en reconnaissant des mots déguisés mais appartenant à (3) un stock international commun. Ceux qui ont une connaissance, même faible, peuvent aider ceux qui n'en ont aucune à communiquer par la (4) médiation entre individus qui n'ont aucune langue en commun. En l'absence d'un médiateur, ces personnes peuvent toutefois parvenir à un certain niveau de communication (5) en mettant en jeu tout leur outillage langagier, en essayant des expressions possibles en différents dialectes ou langues, en exploitant (6) le paralinguistique (mimique, geste, mime, etc.) et en simplifiant radicalement leur usage de la langue » (énumération ajoutée par nos soins). (11)

Le plurilinguisme intègre l'idée de déséquilibre, d'alternance codique, de développement et de dynamisme. Il ouvre d'emblée à une vision en ligne avec les théories de la complexité où différentes composantes, de nature différente, sont en relation et s'influencent l'une l'autre. Il souligne aussi l'interdépendance entre l'individu et le contexte social.

\section{La notion de plurilinguisme: caractéristiques et implications}

Les nouvelles caractéristiques, potentiellement révolutionnaires, de la vision plurilingue se reflètent dans les perspectives théoriques à partir desquelles on cherche à éclairer les mécanismes d'acquisition des langues : la perspective psycho-cognitive, la perspective socio-culturelle et la perspective pédagogique. Regardons-les de plus près pour comprendre leur lien avec la créativité. Dans le domaine des sciences cognitives, un changement considérable s'est produit. Pendant longtemps on a considéré le cerveau humain comme une machine, comme par exemple d'un ordinateur, où chaque partie avait une fonction bien précise et était irremplaçable par une autre. Dans ce sens, en cas de maladie ou d'accident, la fonction reliée à cette partie était compromise à jamais. Cette vision localisationniste des fonctions cérébrales a été de plus en plus remplacée par une conception dynamique où chaque fonction n'est pas liée de manière indissoluble et permanente à une zone cérébrale. Le concept de neuroplasticité (Doidge), et donc l'idée que le cerveau peut se modifier dans le temps, a fait sa percée entrainant un changement radical de notre conception. Non seulement un nouveau paradigme de la connexion est de plus en plus dominant lorsqu'il s'agit d'expliquer le fonctionnement du cerveau (Bickes), mais surtout le cerveau des plurilingues n'est 
plus vu comme la somme de plusieurs cerveaux monolingues, mais plutôt considéré comme un système complexe et unique (Bialystock; Bialystok et Martin ; Perani et al.).

Dans le domaine psychologique, la dimension sociale occupe de plus en plus le devant de la scène. En particulier, sous l'influence des travaux de Lev Vygotsky et contrairement à bien d'autres théories, «la théorie socio-culturelle soutient que le facteurs qui façonnent l'activité mentale ont une origine sociale » (Lantolf et Poehner 52, ma traduction). Par les biais de l'interaction sociale, l'individu bâtit et structure ses connaissances. L'acquisition de la langue se passe, elle aussi, avant tout dans la sphère sociale et est inextricablement liée aux interactions et aux médiations parmi les individus, qui possèdent chacun leur système social complexe, tout en participant aux systèmes (configurations) linguistiquement, socialement et culturellement définis (Lantolf).

Enfin, dans le domaine pédagogique en général et dans celui de la didactique des langues en particulier, la quête du Graal de la méthode la plus efficace qui s'appuyait sur l'une ou l'autre théorie de référence a été remplacée par une vision plus articulée (Mitchell et Vidal), qui implique des démarches et des choix pédagogiques basées sur des principes (Brown), et par une action située (Suchman) adaptée au milieu dans lequel le praticien agit. Une vision nouvelle et complexe des méthodes d'apprentissage des langues a vu le jour, soutenue par le post-method movement (Bell ; Garcia ; Kumaravadivelu).

Ce passage d'un paradigme behaviouriste à une vision de la langue comme cognitivement développée (Garcia et Flores) et socialement construite (Lantolf) ouvre la voie à l'idée que l'individu apprend quand un nouveau processus actif et réflexif se produit et l'information peut être reliée à des connaissances déjà acquises. Les langues maternelles ne sont pas exclues de ce processus : 1'acquisition de chaque (nouvelle) langue modifie la compétence langagière globale des individus et modifie ainsi leur répertoires langagiers. Ceci implique que les erreurs ne sont plus vues comme de simples produits d'interférence, mais aussi, et peut être surtout, comme un moyen de progresser. L'apprentissage des langues est considéré de plus en plus comme un processus non-linéaire, où les connaissances et compétences linguistiques préexistantes sont prises en compte, en même temps que les différents objectifs, conditions et contraintes, ainsi que l'expérience acquise lors des apprentissages langagiers, en lien direct avec l'accomplissement des tâches (Piccardo, «Complessità »). L'exemple du bilinguisme comme tremplin pour l'apprentissage de langues additionnelles est un exemple parlant de la non linéarité de ce processus. 
Il montre, en effet, qu'une véritable synergie entre les différentes langues peut se mettre en place, notamment si la maîtrise de deux langues des bilingues comprend leur composante de littératie (Rauch). Dans cette vision complexe, les apprenants peuvent (et doivent) jouer un rôle très actif. La construction des compétences langagières dépend de (et est améliorée par) une attitude autonome réflexive où les habiletés métacognitives et métalinguistiques sont fondamentales. Elles sont aussi favorisées par une rôle actif de l'apprenant dans l'environnement. La définition de l'apprenant comme «acteur social » proposée par le CECR est très révélatrice de l'importance donnée à l'interaction entre individu et société ainsi qu'à l'agentivité du sujet. Les notions de plurilinguisme et pluriculturalisme permettent de combiner cette pluralité d'éléments et d'habiletés. C'est précisément dans ces espaces, dans cette ouverture entre les langues et cultures, qu'il existe la possibilité d'appréciation esthétique de la langue, d'implication émotionnelle, d'exploration des significats possibles et inventés, de jeu, de créativité.

\section{La créativité, ressource d'une nouvelle vison complexe des langues}

Encore aujourd'hui demeure une idée de multilinguisme comme simple addition de langues au niveau individuel ou de communautés linguistiquement et culturellement différentes qui vivent côte-à-côte. Il y a aussi toujours une forte tendance à privilégier la lingua franca, car « on croit à la transparence fondamentale du langage et donc que la diversité des langues est un facteur négligeable dans l'émergence de nouvelles idées » (Lüdi 3).

Néanmoins, il y a désormais toujours plus de voix contraires qui mettent en avant une certaine opacité des langues (Gajo), en soulignent la valeur et plaident pour la prise en compte du plurilinguisme, pour la « construction, transmission et circulation des connaissances » (Lüdi 3). Dans un terrain qui avait été préparé à prendre en compte la dimension plurielle avec une intérêt grandissant envers les implications et les bénéfices potentiels du bilinguisme et du multilinguisme, le plurilinguisme/pluriculturalisme apparaît comme l'une des notions plus innovantes qui aient été offertes aux domaines scientifiques reliés aux langues, que ce soit la linguistique, la neurolinguistique, la psycholinguistique ou la pédagogie. Cette notion est aussi potentiellement en mesure de favoriser un vrai changement de paradigme. Le passage du multilinguisme au plurilinguisme est bel et bien un passage du simple au complexe. Et la complexité constitue la condition fondamentale pour la créativité (Piccardo, « Créativité »; Csikszentmihalyi). 
Voici une représentation visuelle de la nouvelle notion de plurilinguisme et pluriculturalisme ( $\mathrm{L} 1,2,3 \ldots$...tc. se réfèrent aux langues, et $\mathrm{C} 1,2,3$, etc. aux cultures des individus) :

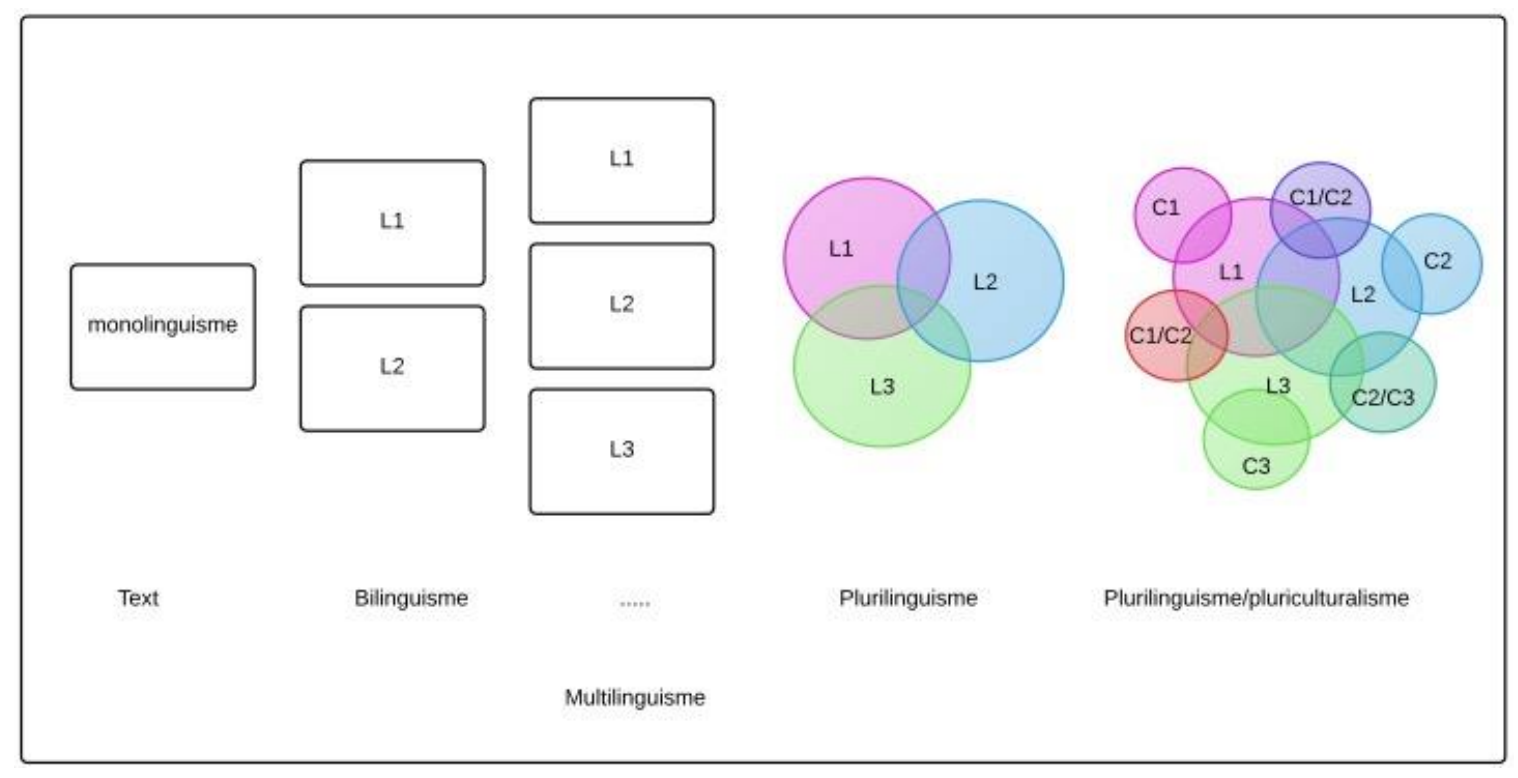

La vision complexe que la notion de plurilinguisme/pluriculturalisme sous-tend, ainsi que son potentiel créatif sont de plus en plus confirmés par la recherche. En psycholinguistique et en neurolinguistique, Ping Li explique que la langue et la cognition interagissent dès les premières phases du développement créant une relation complexe et dynamique entre langue et comportement. Non seulement du point de vue neurophysiologique l'alternance codique fait partie du même système de contrôle exécutif, mais aussi une langue ne peut pas être complètement désactivée quand l'autre est activée, créant ainsi tout une série de situations de compétition et/ou d'interactions entre langues. Les recherches récentes, affirme toujours $\mathrm{Li}$, vont toujours plus dans le sens d'étudier les réseaux du cerveau (la connectivité) en examinant non seulement les zones spécifiques du cerveau, mais aussi les relations spatio-temporelles entre les différentes régions du cerveau pendant le processus cognitif et langagier. Ceci amène à des études longitudinales qui sont nécessaires pour comprendre les changements et les adaptations engendrées par de nouvelles langues ainsi que la compétition créée par la représentation de plusieurs langues/cultures.

Enfin des études très récentes dans le domaine du contact culturel viennent en soutien de la vision avancée par le plurilinguisme/pluriculturalisme. Selon l'une de ces études, l'expérience biculturelle augmente le potentiel créatif, mais ceci est particulièrement évident lorsque les 
individus mélangent leurs identités culturelles. C'est bien cette interpénétration qui semble favoriser la créativité (Saad et al).

La simple exposition à de nombreuses cultures n'est peut-être pas suffisante pour améliorer la créativité comme le suggéraient des recherches précédentes. Plutôt, intégrer les divergences perçues voir brouiller/fusionner les frontières/barrières entre perspectives culturelles distinctes apparaissent comme des actes nécessaires pour favoriser la créativité, ce qui indique les vastes bénéfices cognitifs de pouvoir gérer l'expérience biculturelle (373) (117 Etudiants chinois/américains à l'université de Californie, Davis).

En s'appuyant sur une étude précédente et en étendant ses résultats (Maddux, Adam et Galinsky), une autre recherche a été conduite sur des individus qui ont vécu à l'étranger (quarantetrois participants d'une grande université de Paris) pour voir si l'adaptation à d'autres cultures facilite la créativité. Encore une fois, la conclusion est que la seule exposition à d'autres cultures n'est pas en mesure de produire des changements psychologiques durables reliés à la créativité. Le rôle critique de l'apprentissage, c'est-à-dire de réfléchir à des comportements auxquels on a été habitués pendant longtemps et comment ceux-ci peuvent soudainement avoir différentes fonctions dans différents milieux culturels est fondamental pour stimuler la créativité (Maddux, Adam et Galinsky). Il est impératif de s'engager dans deux cultures différentes simultanément pour stimuler le processus créatif (Maddux, Adam et Galinsky.)

\section{Plurilinguisme et créativité : des synergies}

Les études sur le plurilinguisme et la prise en compte de ses caractéristiques innovantes commencent à faire leur percée au-delà du milieu académique. En Europe, face aux implications de la diversité linguistique et culturelle grandissante de la société globale, à la difficulté d'une application mécanique d'une lingua franca et aux tension créées par une 'simple' homogénéisation culturelle, la pluralité linguistique et culturelle du continent commence à être valorisée et mise en relation avec la créativité. L'année 2009 a marqué une étape clé de ce processus, avec la déclaration de 2009 comme « Année européenne de la créativité et de l'innovation » (Marsh et Hill 1), où l'on affirme que le futur de l'Europe dépend de l'imagination et de la créativité de ses gens. À cette occasion l'Union Européenne a commandité un rapport d'expert visant à examiner une longue série d'études pour vérifier l'existence de liens entre plurilinguisme et créativité. Tout en restant prudents, ils affirment dans les conclusions que les données des trente dernières années montrent 
l'émergence de plusieurs agrégats (clusters) de preuves (6). Les contenus de ces agrégats sont des indicateurs qui montreraient de quelle façon le plurilinguisme «mène à des formes ou des conditions spécifiques pouvant être associés à la créativité » (6).

Les agrégats sont :

1. Souplesse mentale (la pensée flexible) : capacité à activer et à traiter des donnés de catégories multiples non reliées entre elles et de manière simultanée qui serait plus importante lorsque le processus implique plus d'une langue.

2. Capacité à faire face aux difficultés (pensée qui règle les problèmes : les plurilingues ont une capacité supérieure à exercer la fonction exécutive) c'est-à-dire d'interpréter l'information et résoudre les problèmes en décidant de ce qui mérite de l'attention de ce qui n'est pas important. Le contrôle inhibiteur qui permet d'ignorer les distractions donnerait un avantage sur le plan de la créativité. De plus, une vitesse d'exécution plus grande semble liée à la séparation de deux ou plusieurs systèmes linguistiques dans le cerveau.

3. Plus grande habileté métalinguistique. La conscience métalinguistique permet de mieux traiter les données linguistiques, d'acquérir une capacité d'analyse du fonctionnement des langues et de la manière dont on peut l'utiliser pour atteindre un objectif. L'accès aux différentes langues aide à synthétiser les concepts et à les distinguer les uns des autres ce qui peut faire émerger de nouvelles idées.

4. Meilleure faculté d'apprentissage. La personne plurilingue semble avoir plus de facilité à retenir, organiser et stocker l'information, ce qui pourrait se répercuter sur le processus créatif, surtout grâce à l'impact possible de l'interactivité trans-langagière. Il semble par ailleurs que des modifications de l'activité électrique du cerveau se produisent même avec un niveau limité de compétence dans une autre langue.

5. Plus grande facilité de communication interpersonnelle. Le plurilinguisme permettrait d'utiliser de multiples habiletés lors d'une interaction interpersonnelle. Cela pourrait avoir une incidence sur le potentiel créatif.

6. Réduction de l'affaiblissement mental dû au vieillissement. Les modifications de la fonction exécutive et du fonctionnement de la mémoire dus au plurilinguisme pourraient ralentir le déclin de certains processus cognitifs. Donc les personnes plurilingues auraient une « réserve cognitive ». 
Ces éléments sont complétés par une autre contribution parue la même année qui s’appuie sur des recherches en partie différentes. On y trouve cette explication des avantages du plurilinguisme: «it is not unreasonable to propose that the cognitive connection between creativity and plurilingualism is the ability to perceive and produce new units of meaning ... consequently, given high-level plurilingual's increased perceptual awareness, they are likely to gain new insights, create new analogies and experince creative moments in any domain where perception is at work » (Furlong 365).

La connaissance du monde à travers des lentilles différentes et la perception des liens entre les concepts sont potentiellement en mesure de créer des déviations de la norme qui, à leur tour, peuvent faire avancer la perception dans différents domaines. Les plurilingues seraient facilités dans la perception d'analogies créatives et d'associations inattendues. Ces propos sont renforcés aussi par une autre étude très récente qui s'appuie sur une large série de recherches (Kharkhuriu) et qui souligne, entre autres, l'apport du multilinguisme [sic] à la pensée métaphorique. Comme le rappelle Tova Skutnabb-Kangas «[In] an information society [...], the main 'commodities' produced are knowledge and ideas. These are mainly transmitted through language(s) (and visual images). In this kind of information society, those with access to diverse knowledges, diverse information and ideas, will do well, the creativity argument claims » (14).

Ce qui compte est le potentiel que le plurilinguisme a en termes de créativité. Il s'agit de créer des conditions favorables, d'augmenter les chances que des actes créatifs se produisent. Il s'agit en fin de compte d'augmenter le potentiel d'émergence avec toute la richesse que cela comporte. Comme Peter A. Corning le rappelle très clairement, les phénomènes d'émergence impliquent des système complexes (il utilise le terme 'multi-level') (16) qui interagissent avec des systèmes d'ordre inférieur et supérieur et qui ont aussi une influence de cause et effet non seulement horizontaux, mais aussi vers verticaux, vers justement ces autres systèmes. Ceci est complètement cohérent avec le fait qu'à chaque niveau de complexité des propriétés nouvelles apparaissent et que le tout non seulement est plus que la somme des parties mais quelque chose de complètement nouveau comme le faisait remarquer le prix Nobel de physique Phillip Anderson en 1972 dans un article «More is Different » paru dans la revue Science.

Dans ces niveaux de complexité, dans ce quelque chose de nouveau, se creusent des espaces pour la créativité. C'est bien de la possibilité d'un acte créatif qu'il s'agit, de la possibilité de phénomènes de créativité et de productions créatives, et c'est cette justement cette possibilité 
que le plurilinguisme est en mesure d'augmenter. Cependant, pour quelle(s) raison(s) cela se produirait ? Une revue historique du paradigme du plurilinguisme permettra de définir cette notion d'abord, pour ensuite, dans une boucle vertueuse montrer l'interdépendance positive entre plurilinguisme et créativité.

\section{Plurilinguisme créatif ou créativité plurilingue : la dimension pédagogique}

L'ouverture et la souplesse que la notion de plurilinguisme implique, caractérisée par l'entrée de plain-pied d'une dimension plurielle, que ce soit au niveau des langues ou des cultures, et la prise en compte de l'inévitable complexité des idées et des phénomènes présentent un potentiel considérable dans le domaine de l'éducation. Il n'est pas étonnant en fait de voir le foisonnement d'études qui cherchent à comprendre et faire comprendre le passage d'une didactique des langues à une didactique du plurilinguisme. Ce travail présente un intérêt certain, car il favorise la prise de conscience des avantages et des potentialités du plurilinguisme, notamment ceux que nous avons mentionnés plus haut et qui sont listés dans le rapport d'experts de l'Union européenne. Néanmoins, c'est plutôt en renversant la perspective que le changement de paradigme qui est en cours pourra faire sa percée. Il s'agit justement de se donner l'objectif de favoriser un apprentissage créatif, de former à la créativité, ou plutôt d'infuser la créativité dans les pratiques pédagogiques compte tenu que la créativité ne peut pas être une injonction. Il s'agit de créer un terrain favorable, de fournir des occasions pour que les apprenants soient créatifs et de valoriser leur cheminement dans ce sens. Le plurilinguisme devient dans ce cas une ressource, non pas la seule, mais certainement une ressource précieuse, voire incontournable, à laquelle l'apprenant se sent libre de faire appel dans son parcours de construction des connaissances.

Il s'agit de revoir la pédagogie de projet pour l'élargir à - et la nourrir de - la dimension plurilingue. C'est précisément ce que le CECR propose avec ses notions de «perspective actionnelle » et d' «acteur social » (15). Il s'agit d'engager les apprenants dans des tâches proches de la vie réelles, donc a fortiori dans des tâches créatives. Dans la vie réelle, «l'individu fait des expériences et entre en contact avec un nombre croissant d'autres individus et cela contribue à définir son identité. L'apprenant prend conscience de ses propres connaissances et compétences et les utilise dans et pour l'action sociale. À son tour cette utilisation sociale, cette mise en commun, lui permet d'avoir un retour, une rétroaction, qui l'aidera à avancer dans la construction de ses connaissances et compétences. Il s'agit de faire quelque chose pour apprendre et non pas 
d'apprendre pour faire quelque chose » (Piccardo, Du communicatif, 19). Avec cette vision, nous nous rapprochons de la logique que Mihaly Csikszentmihalyi nous propose en 1988 dans son system model de la créativité. Ce modèle représente la créativité comme résultant « des interactions d'un système composé de trois éléments : une culture dotée de règles symboliques, une personne qui introduit une nouveauté dans ce domaine symbolique et des experts qui reconnaissent et valident l'innovation » (12).

Ce n'est pas un hasard que l'un des arguments majeurs pour soutenir le plurilinguisme soit celui qui encourage la biodiversité, ce que Skutnabb-Kangas appelle «the heritage and biodiversity arguments » qui voit dans la diversité linguistique et culturelle the «storehouse of historically developed knowledges » (13). Csikszentmihalyi fait un parallèle intéressant entre le processus de la créativité et celui la génétique. Le processus de créativité génère beaucoup d'idées et de production nouvelles, et ensuite c'est l'environnement qui opère une sélection. Or, comme nous le savons bien, la richesse et la variété génétique jouent un rôle fondamental et très positif dans l'évolution. De la même manière, le plurilinguisme et l'attention portée à la diversité multiplient les chances d'un travail créatif qui à son tour favorise la prise de conscience de la valeur de cette même diversité. Une mise en œuvre de l'approche actionnelle à travers des tâches créatives qui ouvrent au plurilinguisme et aux trajectoires de chaque acteur social peut favoriser cette boucle vertueuse.

\section{Conclusion}

Etudier la créativité à l'aide de la théorie du plurilinguisme nous permet de saisir comment un terme unique peut comprendre plusieurs conceptions et perspectives différentes. Nous avons retrouvé dans l'analyse du plurilinguisme plusieurs affinités avec les éléments individués par la recherche comme capables de favoriser la créativité : de la prise de risque, à la nécessité d'un effort en vue de la maitrise d'un domaine disciplinaire, de l'importance du milieu à la capacité à saisir et transgresser les frontières entre domaines. Justement, les plurilingues seraient plus capables de percevoir les frontières et de les dépasser, donc de créer des in-between spaces. Mais ce n'est pas une vision multilingue qui permet de faire vivre ces espaces et qui permet aux individus de les prendre en compte/se les approprier/les habiter. Ceci est seulement possible si on va vers le plurilinguisme et vers le pluriculturalisme. 
Alors que souvent l'enseignement de plusieurs langues se fait selon un paradigme de la simplicité en prenant soin de garder les langues bien distinctes, ce sont précisément tous les phénomènes de métissage, de déséquilibre, de dépassement de la norme, ces « in-between spaces » les espaces intermédiaires, qui comme nous le rappelle Georges Lüdi (18) peuvent être dans l'interaction sociale mais aussi dans l'esprit d'un locuteur plurilingue et pluriculturel même. Tout ce qui est à la base du plurilinguisme semble en mesure de créer les conditions favorables à la créativité. Csickszentmihalyi dit que «nous naissons tous avec deux séries d'instructions contradictoires: une tendance conservatrice qui comprend les instincts d'auto-préservation, d'auto-accroissement et d'économie de notre énergie, et une tendance expansive faite des instincts d'explorations, du plaisir de la nouveauté, du risque - la curiosité qui produit la créativité appartient à cette série » (17). L'utilisation d'une lingua franca, fait certes partie de la première série, mais aussi un « simple » multilinguisme, une accumulation linéaire des langues, des cultures, des communautés, un laisser-faire par la globalisation. Une forme de «agency », d'agentivité, et un immense travail de toutes les parties prenantes pour aller vers le plurilinguisme et le pluriculturalisme, dans une perspective complexe peut au contraire augmenter énormément les chances que la créativité se développe à tous les niveaux.

Une métaphore puissante a été choisie pour décrire la créativité (Johnson-Laird), celle de la jam session qui constitue en langue un espace potentiellement créatif. Comme dans une jam session, il faut maitriser son domaine, mais il faut aussi savoir prendre des risques, c'est nouveau et imprévisible. L'évaluation et l'idéation doivent procéder en parallèle, le processus d'émergence a lieu dans l'interaction entre l'individu et le groupe, mais l'ensemble qui en résulte est plus important que la simple somme de ses parties.

\section{Bibliographie}

Anderson, Philip W. «'More is Different' : Broken Symmetry and the Nature of the Hierarchical Structure of Science ». Science 177 (1972) : 393-96.

Baker, Colin. Key issues in Bilingualism and Bilingual Education. Clevedon: Multilingual Matters, 1988.

Bell, David M. «Method and Postmethod : Are they really so Incompatible?». TESOL Quarterly $37.2(2003): 325-336$. 
Bialystok, Ellen. Bilingualism in Development : Language, Literacy, and Cognition, Cambridge: Cambridge UP, 2001.

--- et Martin Michelle M. «Attention and Inhibition in Bilingual Children : Evidence from the Dimensional Change Card Sort Task ». Developmental Science 7.3 (2004) : 325-39.

Bickes, Hans. «Bilingualismus, Mehrsprachigkeit und mentales Lexikon Evolutionsbiologische, soziokulturelle und kognitionswissenschaftliche Perspektiven ». Fremdsprachen lehren und lernen 33 (2004) : 27-51.

Brown, H. Douglas. Principles of Language Learning and Teaching. New York: Pearson Education, 2007.

Canagarajah, Suresh. «The place of World Englishes in Composition : Pluralization Continued ». College Composition and Communication 57 (2006) : 586-619.

--- et Liynage, Indika. «Lessons from Pre-colonial Multilingualism ». The Routledge Handbook of Multilingualism. Dir. Marilyn Martin-Jones, Adrian Blackledge et Angela Creese. London/New York: Routledge, 2012. 49-65.

Castles, Stephen et Mark J. Miller. The Age of Migration. $3^{\mathrm{e}}$ éd. Basingstoke/New York : Palgrave Mcmillan, 2003.

Chomsky, Noam. Aspects of the Theory of Syntax. Cambridge, MA: MIT P, 1965.

Conseil de l'Europe. Cadre européen commun de référence pour les langues (CECR). Paris : Hatier-Didier, 2001.

Cook, Vivian J. «The Poverty-of-the-Stimulus Argument and Multi-Competence ». Second Language Research 7.2 (1991) : 103-117.

---. «The Goals of ELT : Reproducing Native-Speakers or Promoting Multi-Competence among Second Language Users? ». Handbook on English Language Teaching. Dir. Jim Cummins et Chris Davison. Dordrecht : Kluwer, 2007.

Corning, Peter A. «The Re-emergence of 'Emergence' : A Venerable Concept in Search of a Theory ». Complexity 7.6 (2002) : 18-30

Coste, Daniel, Danièle Moore et Geneviève Zarate. Compétence plurilingue et pluriculturelle. Vers un Cadre européen commun de référence pour l'enseignement et l'apprentissage des langues vivantes. Études préparatoires. Strasbourg : Conseil de l'Europe, 1997. 
Council of Europe (2006). Plurilingual and Intercultural Education as a Project. http://www.coe.int/t/dg4/linguistic/Source/LE_texts_Source/EducPlurInter-Projet_en.pdf. Consulté le 20-04-2015.

Csikszentmihalyi, Mihaly. «Society, Culture and Person : A Systems View of Creativity ». The Nature of Creativity. Dir. Robert J. Sternberg. Cambridge, UK: Cambridge UP, 1988. 4761

---. La créativité. Psychologie de la découverte et de l'invention. Paris : Robert Laffont, 2006 (1996).

Darcy. Natalie T. «A Review of the Literature on the Effects of Bilingualism upon the Measurement of Intelligence ». Journal of Genetic Psychology 82 (1953) : 21-57.

---. «Bilingualism and the Measurement of Intelligence : Review of a Decade of Research ». Journal of Genetic Psychology 103 (1963) : 259-282.

Defert, Jean-Jacques. «Appréhender la complexité. Enjeux et raisonances dans le domaine culturel ». International Journal of Canadian Studies / Revue internationale d'études canadiennes 45-46 (2012) : 307-329.

Doidge, Norman. The Brain that Changes Itself. New York : Penguin, 2007.

Furlong, Aine. "The Relation of Plurilingualism/Culturalism to Creativity : A Matter of Perception ». International Journal of Multilingualis 6/4 (2009) : 343-368.

Gajo, Laurent. «Types de savoirs dans l'enseignement bilingue : problématicité, opacité, densité ». Education et sociétés plurilingues 20 (2006) : 75-87.

García, Ofelia. Bilingual Education in the 21st Century : A Global Perspective. Malden, MA: Wiley-Blackwell, 2009.

--- et Nelson Flores. « Multilingual Pedagogies ». The Routledge Handbook of Multilingualism.

Dir. Marilyn Martin-Jones, Adrian Blackledge et Angela Creese. London/New York: Routledge, 2012. 232- 246.

Gogolin, Ingrid. Der monolinguale Habitus der multilingualen Schule [The monolingual habitus of multilingual school]. Münster: Waxmann, 1994.

Goldstein, Jeffrey. «Emergence as a Construct : History and Issues ». Emergence. A Journal of Complexity Issues in Organizations and Management 1.1 (1999) : 59-72. 
Hakuta Kenji et Raphael M. Diaz. «The Relationship between Degree of Bilingualism and Cognitive Ability : A Critical Discussion and some New Longitudinal Data ». Children's Language 5 (1985) : 320-344.

Ianco-Worrall et Anita D. «Bilingualism and Cognitive Development ». Child Development 43 (1972) : 1390-1400.

Jensen, J. Vernon. «Effects of Childhood Bilingualism ». Elementary English 39 (1962) : 132-43.

Johnson-Laird et Philip N. «How Jazz Musicians Improvise ». Music Perception 19.3 (2002) : 415-427.

Jørgensen, J. Normann. «Polylingual Languaging Around and Among Children and Adolescents ». International Journal of Multilingualism 5.3 (2008) : 161-176.

Juignet, Patrick. « Le concept d'émergence ». Philoscience.com (2010). http://www.philosciences.com/General/Emergence.html

Kharkhuriu, Anatoliy. Multilingualism and Creativity. Bristol: Multilingual Matters, 2012.

Kumaravadivelu, B. Kumar. «Toward a Postmethod Pedagogy ». TESOL Quarterly 35.4 (2001) : 537-560.

Lantolf, James. «The Sociocultural Approach to Second Language Acquisition : Sociocultural Theory, Second Language Acquisition, and Artificial L2 Development». Alternative Approaches to Second Language Acquisition. Dir. Dwight Atkinson. NY: Routledge, 2011. 24-47.

Lantolf, J. et Matthew Poehner. Sociocultural Theory and the Pedagogical Imperative in L2 Education : Vygotskian Praxis and the Research/Practice Divide. NY: Routledge, 2014.

Li, Ping. « Computational Modeling of Bilingualism. A Special Issue of Bilingualism ». Language and Cognition 16. 2 (2013) : 241-366.

Lüdi, Georges. «Le plurilinguisme comme source de créativité et d'innovation dans le monde du travail ». Vox Romanica 69 (2010) : 1-24.

Maddux, William W., Hajo Adam et Adam D. Galinsky. «When in Rome . . Learn Why the Romans Do What They Do : How Multicultural Learning Experiences Facilitate Creativity ». Personality and Social Psychology Bulletin 36. 6 (2010) : 731-741.

Makoni, S. et Alistair Pennycook. Disinventing and Reconstituting Languages. Clevedon : Multilingual Matters, 2007.

Marsh, David. et Richard Hill, dir. Étude sur la contribution du multilinguisme à la créativité. 
Rapport final. Commission européenne. Direction générale Éducation et Culture, 2009.

Disponible en ligne

http://eacea.ec.europa.eu/llp/studies/documents/study_on_the_contribution_of_multilingu alism_to_creativity/final_report_fr.pdf

Mitchell, Cheryl Brown et Kari Ellington Vidal. «Weighing the Ways of the Flow : Twentiethcentury Language Instruction ». The Modern Language Journal 85.1 (2001) : 26-38.

North, Brian. The CEFR in Practice. Cambridge : Cambridge UP, 2014.

Peal, Elizabeth et Wallace E. Lambert. «The Relation of Bilingualism to Intelligence ». Psychological Monographs 76.27 (1962) : 1-23.

Perani, Daniela et al.. «The Role of Age of Acquisition and Language Usage in Early, Highproficient Bilinguals : An fMRI Study during Verbal Fluency ». Human Brain Mapping 19.3 (2003) : 170-182.

Piccardo, Enrica «Complessità e insegnamento delle lingue straniere: ripensare un paradigma ». RILA (Rassegna Italiana di Linguistica Applicata) 37.2-3 (2005) : 75-92.

---. «L'enseignant : un stratège de la complexité ». La formation des enseignants en Europe : Approche comparative. Dir. Gilles Baillat, Daniel Niclot et Dominique Ulma. Bruxelles : De Boeck, 2010. 79-97.

---. «Plurilingualism and Curriculum Design : Towards a Synergic Vision ». TESOL Quarterly 47.3 (2013) : 600-614.

---. Du communicatif à l'actionnel : un cheminement de recherche. Curriculum Services Canada/Ontario Ministry of Education, 2014a. http://www.curriculum.org/storage/241/1410360061/TAGGED_DOCUMENT_\%28CSC 605_Research_Guide\%3B_French\%29_03.pdf

---. «The Impact of the CEFR on Canada's Linguistic Plurality : A Space for Heritage Languages?». Rethinking Heritage Language Education. Dir. Peter Trifonas et Themistocles Aravossitas. Cambridge: Cambridge UP, 2014b. 183-212.

---. «Créativité et complexité : quels modèles, quelles conditions, quels enjeux ? ». La créativité en éducation et en formation. Perspectives théoriques et pratiques. Dir. Isabelle Capron Puozzo. Bruxelles : De Boeck, sous presse.

---, et al. Parcours d'évaluation, d'apprentissage et d'enseignement à travers le CECR. Strasbourg : Conseil de 1'Europe, 2011. 
Rauch, Dominique. «Effects of Biliteracy on Third Language Reading Proficiency, the Example of Turkish-German Bilinguals ». Plurilingual Education. Policies - Practices - Language Development. Dir. Patrick Grommes et Adelheid Hu. Amsterdam : Benjamins, 2014. 199218

Saad, Carmel et al. «Multiculturalism and Creativity : Effects of Cultural Context, Bicultural Identity, and Cognitive Fluency ». Social Psychological and Personality Science 4 (2013) : 368-374.

Sawyer, Robert Keith. Explaining Creativity. The science of Human Innovation. Oxford: Oxford UP, 2012 (2006).

---. « The Emergence of Creativity ». Philosophical Psychology 12.4 (1999) : 447-469.

Skutnabb-Kangas, Tova. Why Should Linguistic Diversity Be Maintained and Supported in Europe? Some Arguments. Guide for the Development of Language Education Policies in Europe from Linguistic Diversity to Plurilingual Education. Strasbourg: Council of Europe, 2002. http://www.coe.int/t/dg4/linguistic/Source/Skutnabb-KangasEN.pdf

Suchman, Lucy A. Plans and Situated Actions : The Problem of Human-Machine Communication. Cambridge: Cambridge UP, 1987.

Vertovec, Steven. «Super-Diversity and its Implications ». Ethnic and Racial Studies 29.6 (2007) : 1024-54. 